\title{
A novel and specific fluorescence reaction for uracil
}

\author{
Takayuki Shibata ${ }^{a}$, Shin-ya Kawasaki ${ }^{a}$, Jun-ya Fujita ${ }^{a}$, Tsutomu Kabashima ${ }^{a}$, \\ Masaaki Kai ${ }^{\mathrm{a}, \mathrm{b}, *}$
}

${ }^{a}$ Faculty of Pharmaceutical Sciences, Graduate School of Biomedical Sciences, Nagasaki University, 1-14 Bunkyo-Machi, Nagasaki 852-8521, Japan

${ }^{b}$ Global Center of Excellence Program, Nagasaki University

*Corresponding author. Tel. and fax: +81-95-819-2438; E-mail: ms-kai@nagasaki-u.ac.jp.

\begin{abstract}
Facile and specific methods to quantify a nucleobase in biological samples are of great importance for diagnosing disorders in nucleic acid metabolism. In the present study, a novel fluorogenic reaction specific for uracil has been developed. The reaction was carried out in an alkaline medium containing benzamidoxime and $\mathrm{K}_{3}\left[\mathrm{Fe}(\mathrm{CN})_{6}\right]$ with heating for $2.0 \mathrm{~min}$. Under the optimum reaction conditions, strong fluorescence was produced only from uracil, not from other many biogenic compounds tested such as cytosine, thymine, adenine, guanine, nucleobases, nucleosides, nucleotides, amino acids, saccharides, creatine, creatinine and urea. The sensitivity of this method was compared with a known fluorogenic reaction using phenacylbromide which does not react with uracil but reacts with cytosine, adenine and their analogues. The proposed uracil-specific reaction showed approximately 400 fold higher sensitivity than the phenacylbromide reaction. The lower detection limit of uracil by the present method was $100 \mathrm{pmol} \mathrm{mL}^{-1}$, and a good linearity of the calibration curve was obtained up to $100 \mathrm{nmol} \mathrm{mL}^{-1}$ uracil. Due to its high sensitivity and specificity, the quantitative determination of uracil was possible by the proposed fluorimetric method.
\end{abstract}

Keywords: Fluororescence reaction; Benzamidoxime; Uracil specificity; High sensitivity; Pyrimidine metabolism. 


\section{Introduction}

Uracil is metabolised to $\beta$-alanine via a three-step catabolic pathway which includes the rate-determining step from uracil to 5, 6-dihydrouracil by dihydropyrimidine dehydrogenase (DPD) in a mammalian body, as shown in Fig. 1 [1-4]. On the other hand, fluorinated uracil derivatives such as 5-fluorouracil (5-FU) and its pro-drug, 5-fluoro-1-(tetrahydro-2-furanyl)2,4-(1H,3H)-pyrimidine-dione (tegafur), can be potent anticancer drugs because of the inhibition of DNA replication [5]. Since 5-FU also is catabolised by DPD [6-8], a loss of DPD activity causes an accumulation of 5-FU in blood after administration, and leads to severe side effects including death (Fig. 1, right side) [9-14]. Moreover, inherited disorders in the degradation pathway of pyrimidine are the major criteria of the inborn errors of pyrimidine metabolism, and are known to show a variety of symptoms such as convulsion, mental retardation and microcephaly [15]. Quantification of uracil in blood and urine is of great importance to identify the level of pyrimidine metabolism, especially for the diagnosis of DPD deficiency.

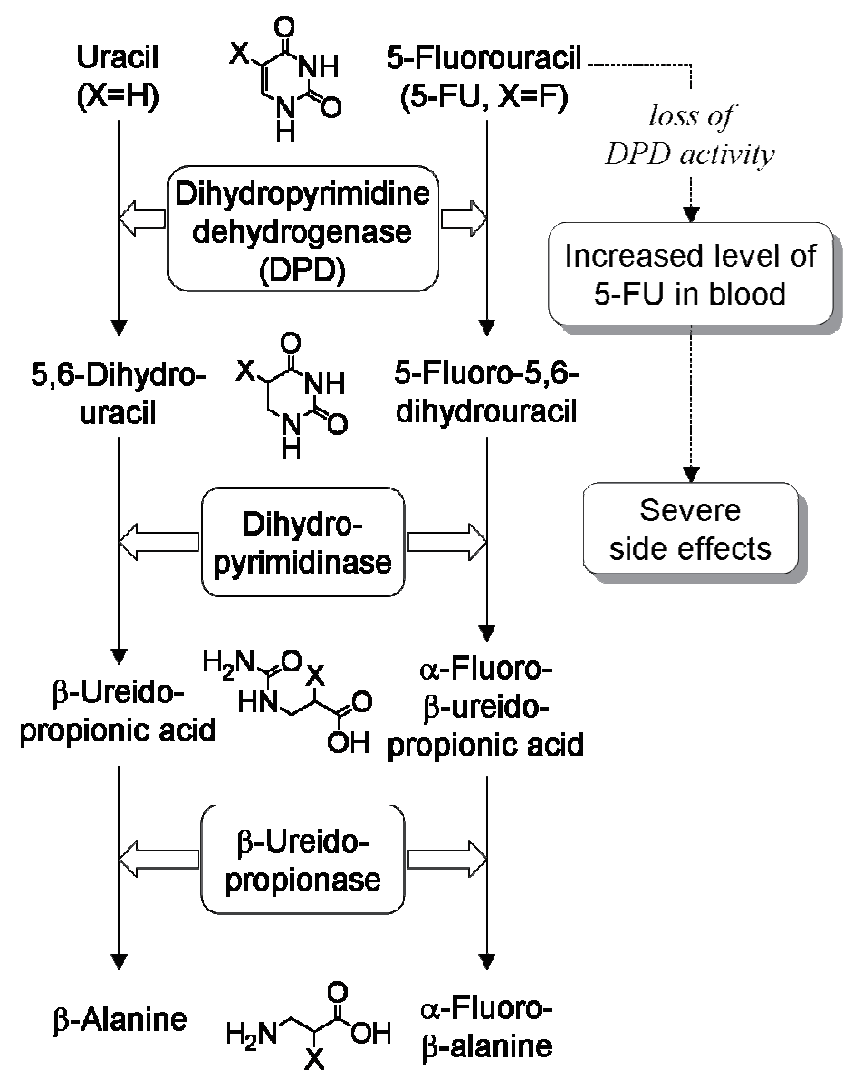

Fig. 1. Catabolic pathway of uracil and 5-FU.

Methods for the quantification of uracil in biological samples have utilized high-performance liquid chromatography (HPLC) and mass spectrometry (MS) e.g. tandem HPLC method with column switching [16], HPLC/tandem MS system [17], gas 
chromatography/MS system [18] and radiometric HPLC [19-22]. Although these methods are superior for the determination of a complex sample, the instrumentations need high cost for the analysis. Recently, Mattison et al. demonstrated a non-chromatographic method for the analysis of the level of uracil degradation, in which $2-\left[{ }^{13} \mathrm{C}\right]$-labelled uracil was administrated and the catabolised ${ }^{13} \mathrm{CO}_{2}$ in exhaled breath sample was measured by infrared spectrometry [23]. This method is convenient for evaluating the metabolic function of uracil. However, the stage at which the error occurs in pyrimidine catabolism cannot be identified since three enzymes are involved in the digestion of uracil to $\mathrm{CO}_{2}$.

Therefore, a facile and high-throughput technique to quantify uracil has been highly demanded. Fluorescence (FL) reaction is one of the widely-used methodologies for sensitive, facile and simultaneous detection of multiple samples in a multi-well plate. In this article, we describe a novel FL-deriving reaction specific for uracil for the first time. This reaction was conducted by heating uracil with benzamidoxime $(\mathrm{BAO})$ and $\mathrm{K}_{3}\left[\mathrm{Fe}(\mathrm{CN})_{6}\right]$ in a $\mathrm{KOH}$ solution. The proposed method could sensitively measure uracil with a lower detection limit of $50 \mathrm{fmol}$ per an injection volume by HPLC and $100 \mathrm{pmol} \mathrm{mL}^{-1}$ in the reaction mixture by a spectrofluorimeter. To highlight the specificity of the current reaction, the mechanism of the reaction is also discussed.

\section{Experimental}

\subsection{Chemicals}

Benzamidine hydrochloride and $\mathrm{K}_{3}\left[\mathrm{Fe}(\mathrm{CN})_{6}\right]$ were purchased from Nacalai Tesque (Kyoto, Japan). The other oxidising agents were from Wako (Osaka, Japan). BAO and benzohydroxamic acid were obtained from TCI (Tokyo, Japan). The other BAO-related chemicals were from Sigma-Aldrich (MO, USA). All chemicals were of analytical or guaranteed reagent grade and were used without further purification. Water was purified on a Milli-Q system WR 600 A from Millipore (Molsheim, France).

\subsection{Procedure recommended for the FL reaction of uracil}

Uracil, $\mathrm{BAO}, \mathrm{K}_{3}\left[\mathrm{Fe}(\mathrm{CN})_{6}\right]$ and $\mathrm{KOH}$ were weighed in different flasks and water was added to them to prepare for four stock solutions. To a $1.5-\mathrm{mL}$ tube was added $250 \mu \mathrm{L}$ of each reactant in the order of $4.0 \times 10^{-4}-4.0 \mu \mathrm{mol} \mathrm{mL} \mathrm{m}^{-1}$ uracil, $4.0 \mathrm{mmol} \mathrm{L}^{-1} \mathrm{BAO}, 8.0 \mathrm{mmol} \mathrm{L}^{-1}$ $\mathrm{K}_{3}[\mathrm{Fe}(\mathrm{CN})]_{6}$ and $4.0 \mathrm{~mol} \mathrm{~L}^{-1} \mathrm{KOH}$. The mixture was then heated at $90{ }^{\circ} \mathrm{C}$ for $2.0 \mathrm{~min}$, followed by cooling in an ice bath for $2.0 \mathrm{~min}$ to stop the reaction.

\subsection{FL detection}

The relative FL intensity of the final reaction mixture in a quartz cell $(10 \times 4 \mathrm{~mm})$ was measured with an FP-6300 spectrofluorimeter (Jasco, Tokyo, Japan) at excitation and 
emission wavelengths of $320 \mathrm{~nm}$ and $410 \mathrm{~nm}$, respectively with both the band widths of $5 \mathrm{~nm}$. The identification of the fluorescent derivative of the nucleobase was carried out on a reversed-phase HPLC system, consisting of a PU-2089 quaternary gradient pump, an FP-920 FL detector, and an UV-970 detector (Jasco, Tokyo, Japan). The separation of the fluorescent derivative was performed on a TSKgel ODS-80Ts reversed-phase column $(250 \times 4$.6-mm ID) (TOSOH, Tokyo, Japan) with an isocratic elution employing $30 \%(\mathrm{v} / \mathrm{v})$ methanol in water containing $10 \mathrm{mmol} \mathrm{L}^{-1}$ triethylammonium acetate $(\mathrm{pH} 7.0)$ at a constant flow rate of $1.0 \mathrm{~mL}$ $\min ^{-1}$.

\subsection{Phenacylbromide reaction}

The reaction was conducted according to the reported procedure with a slight modification [24]. To a $200-\mu \mathrm{L}$ aqueous solution of cytosine in a $1.5-\mathrm{mL}$ tube was added $600 \mu \mathrm{L}$ of acetic acid-acetonitrile-water solution $(1: 80: 19, \mathrm{v} / \mathrm{v})$ followed by adding $200 \mu \mathrm{L}$ of $12.5 \mathrm{mmol} \mathrm{L}^{-1}$ phenacylbromide in acetonitrile. The reaction mixture was heated at $80{ }^{\circ} \mathrm{C}$ for $45 \mathrm{~min}$. The FL intensity was then measured in the same manner as described above, except for an excitation and emission wavelengths of $320 \mathrm{~nm}$ and $370 \mathrm{~nm}$, respectively.

\subsection{Large scale synthesis of FL product}

To a $25-\mathrm{mL}$ portion of $1.0 \mu \mathrm{mol} \mathrm{mL} \mathrm{mL}^{-1}$ uracil $(2.8 \mathrm{mg}, 25 \mu \mathrm{mol})$ was added $25 \mathrm{~mL}$ each of $4.0 \mathrm{mmol} \mathrm{L} \mathrm{L}^{-1}$ 4-trifluoromethylbenzamidoxime $(20.4 \mathrm{mg}, 0.1 \mathrm{mmol}), 8.0 \mathrm{mmol} \mathrm{L}^{-1}$ $\mathrm{K}_{3}\left[\mathrm{Fe}(\mathrm{CN})_{6}\right](65.9 \mathrm{mg}, 0.2 \mathrm{mmol})$ and $2.0 \mathrm{~mol} \mathrm{~L}^{-1} \mathrm{KOH}$. All the chemicals were dissolved in $\mathrm{H}_{2} \mathrm{O}$. The mixture was heated at $100^{\circ} \mathrm{C}$ for $10 \mathrm{~min}$, at which point the reaction was cooled in an ice bath. Four identical reactions were conducted simultaneously, and the resulting reaction mixtures $(4 \times 100 \mathrm{~mL})$ were combined. Potassium chloride was added to the mixture until it reached saturation, and the remaining precipitate of $\mathrm{KCl}$ was filtered off. An aqueous layer was extracted with ethylacetate (4 times with $100 \mathrm{~mL}$ ), and the combined organic layer was dried over $\mathrm{Na}_{2} \mathrm{SO}_{4}$, filtered and evaporated to dryness to give $45 \mathrm{mg}$ of the crude product. The above procedure was repeated for 13 times and the resulting crude products were combined $(826 \mathrm{mg}$ ). Purification by silica gel column chromatography (ethylacetate : methanol $=9: 1$ to $8: 2$ to $7: 3)$ gave the analytically pure product as a pale blue powder $(25 \mathrm{mg}, 6 \%)$.

\section{Results and discussion}

\subsection{Reaction of uracil and cytosine with BAO.}

In our preliminary studies to develop a new fluorogenic reaction specific for uracil, we found that uracil emitted blue FL when it was heated with $\mathrm{BAO}$ and $\mathrm{K}_{3}\left[\mathrm{Fe}(\mathrm{CN})_{6}\right]$ in an alkaline aqueous solution. When uracil was replaced with cytosine, weak blue FL was observed. The other three bases (adenine, guanine and thymine), uridine and 2'-deoxyuridine were all fluorescent-inactive. BAO is a non-fluorescent compound and has not ever been 
utilized for FL reactions. Furthermore, there was no FL reaction specific for uracil. Thus, the discovery of this unique FL reaction encouraged us to further investigate characteristics of the reaction.

In order to increase the specificity for uracil, this reaction conditions were investigated using $250 \mathrm{nmol} \mathrm{mL} \mathrm{mL}^{-1}$ each of uracil and cytosine (Fig. 2). FL intensities were produced from uracil and cytosine, and both increased with the concentration of $\mathrm{K}_{3}\left[\mathrm{Fe}(\mathrm{CN})_{6}\right]$ up to $2 \mathrm{mmol}$ $\mathrm{L}^{-1}$ (Fig. 2a). However, the FL was dramatically decreased when the concentration of the oxidant was further increased. Influence of the BAO concentration (Fig. 2b) indicated different pattern from the results for the $\mathrm{K}_{3}\left[\mathrm{Fe}(\mathrm{CN})_{6}\right]$ concentration. No FL was initially generated up to $0.75 \mathrm{mmol} \mathrm{L}^{-1}$ of the BAO concentration. After further increase of the BAO concentration, then dramatic increases of the FL intensity were observed.

Although similar patterns for the uracil and cytosine conditions were obtained by the reaction temperature (Fig. 2d) as well as the concentrations of $\mathrm{K}_{3}\left[\mathrm{Fe}(\mathrm{CN})_{6}\right]$ and $\mathrm{BAO}, \mathrm{KOH}$ concentration (Fig. 2c) and reaction time (Fig. 2e) showed different patterns between the uracil and cytosine reactions. Non FL was produced when the reaction was conducted without $\mathrm{KOH}$, however the FL was generated by increasing the concentration of $\mathrm{KOH}$. In case of uracil, a maximum FL intensity was obtained when the $\mathrm{KOH}$ concentration was $0.5 \mathrm{~mol} \mathrm{~L}^{-1}$ or greater, whilst $0.025 \mathrm{~mol} \mathrm{~L}^{-1} \mathrm{KOH}$ was found to be the optimum for cytosine. In difference of the reaction time, the FL of cytosine was not produced until 2 min (Fig. 2e). In case of uracil, however its FL intensity gradually increased with reaction time and reached the maximum at $5 \mathrm{~min}$. These results might be attributed to the difference in the reaction mechanism between uracil and cytosine.

In order to investigate a process of the FL production, each reaction mixture for uracil and cytosine was analysed by HPLC with FL detection (Fig. $2 \mathrm{f}$ and $\mathrm{g}$ ). The HPLC profile for the reaction mixture of uracil showed a single FL peak, and a lower detection limit of uracil was approximately 50 fmol per injection $(\mathrm{S} / \mathrm{N}=3)$. On the other hand, two small FL peaks appeared in case of cytosine, and one of two peaks was at the same retention time as that of the uracil product. The results suggested that the initiation of the reaction with cytosine relies on the decomposition of cytosine, essentially deamination at the 4-position of cytosine to uracil. 
(a)

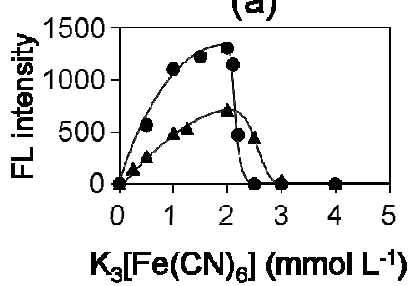

(c)

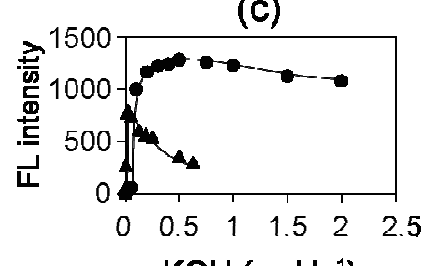

$\mathrm{KOH}\left(\mathrm{mol} \mathrm{L}^{-1}\right)$

(e)

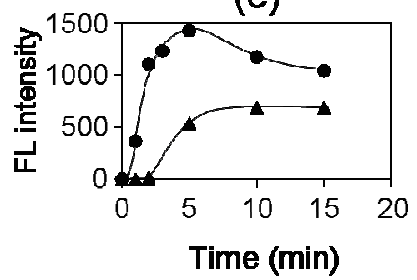

(b)

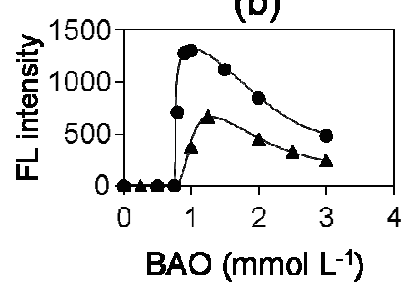

(d)

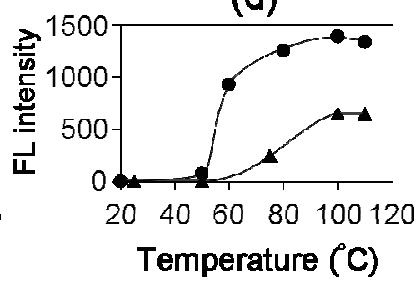

(g)

$(f)$

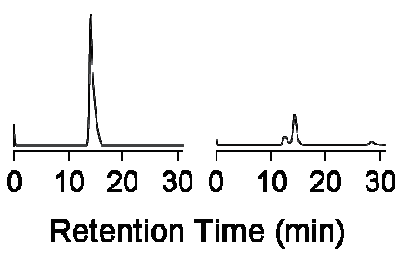

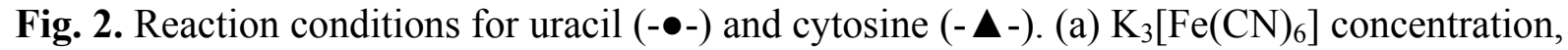
(b) BAO concentration, (c) $\mathrm{KOH}$ concentration, (d) reaction temperature and (e) reaction time. The reaction mixtures with (f) uracil and (g) cytosine were individually separated by HPLC with FL detection.

Table 1 Effect of the order of reagent addition on FL intensity.

\begin{tabular}{cccc}
\hline The order of reagent addition $^{\mathrm{a}}$ & RFI $^{\mathrm{d}}$ & The order of reagent addition $^{\mathrm{a}}$ & RFI $^{\mathrm{d}}$ \\
\hline $\mathrm{U} \rightarrow \mathrm{B} \rightarrow \mathrm{F} \rightarrow \mathrm{K}$ & 100 & $\mathrm{~F} \rightarrow \mathrm{K} \rightarrow \mathrm{U} \rightarrow \mathrm{B}$ & 76 \\
$\mathrm{U} \rightarrow \mathrm{B} \rightarrow \mathrm{K} \rightarrow \mathrm{F}$ & 91 & $\mathrm{~F} \rightarrow \mathrm{K} \rightarrow \mathrm{B} \rightarrow \mathrm{U}$ & 24 \\
$\mathrm{U} \rightarrow \mathrm{F} \rightarrow \mathrm{B} \rightarrow \mathrm{K}$ & 98 & $\mathrm{~K} \rightarrow \mathrm{U} \rightarrow \mathrm{B} \rightarrow \mathrm{F}$ & 90 \\
$\mathrm{U} \rightarrow \mathrm{F} \rightarrow \mathrm{K} \rightarrow \mathrm{B}$ & 95 & $\mathrm{~K} \rightarrow \mathrm{U} \rightarrow \mathrm{F} \rightarrow \mathrm{B}$ & 91 \\
$\mathrm{U} \rightarrow \mathrm{K} \rightarrow \mathrm{B} \rightarrow \mathrm{F}$ & 92 & $\mathrm{~K} \rightarrow \mathrm{B} \rightarrow \mathrm{U} \rightarrow \mathrm{F}$ & 92 \\
$\mathrm{U} \rightarrow \mathrm{K} \rightarrow \mathrm{F} \rightarrow \mathrm{B}$ & 92 & $\mathrm{~K} \rightarrow \mathrm{B} \rightarrow \mathrm{F} \rightarrow \mathrm{U}$ & 22 \\
$\mathrm{~B} \rightarrow \mathrm{U} \rightarrow \mathrm{F} \rightarrow \mathrm{K}$ & 54 & $\mathrm{~K} \rightarrow \mathrm{F} \rightarrow \mathrm{U} \rightarrow \mathrm{B}$ & 80 \\
$\mathrm{~B} \rightarrow \mathrm{U} \rightarrow \mathrm{K} \rightarrow \mathrm{F}$ & 64 & $\mathrm{~K} \rightarrow \mathrm{F} \rightarrow \mathrm{B} \rightarrow \mathrm{U}$ & 28 \\
$\mathrm{~B} \rightarrow \mathrm{F} \rightarrow \mathrm{U} \rightarrow \mathrm{K}$ & 104 & $(\mathrm{U}+\mathrm{B}) \rightarrow(\mathrm{F}+\mathrm{K})^{\mathrm{b}}$ & 89 \\
$\mathrm{~B} \rightarrow \mathrm{F} \rightarrow \mathrm{K} \rightarrow \mathrm{U}$ & 14 & $(\mathrm{U}+\mathrm{F}) \rightarrow(\mathrm{B}+\mathrm{K})^{\mathrm{b}}$ & 92 \\
$\mathrm{~B} \rightarrow \mathrm{K} \rightarrow \mathrm{U} \rightarrow \mathrm{F}$ & 109 & $(\mathrm{U}+\mathrm{K}) \rightarrow(\mathrm{B}+\mathrm{F})^{\mathrm{b}}$ & 92 \\
$\mathrm{~B} \rightarrow \mathrm{K} \rightarrow \mathrm{F} \rightarrow \mathrm{U}$ & 56 & $(\mathrm{U}+\mathrm{B}+\mathrm{F}) \rightarrow \mathrm{K}^{\mathrm{c}}$ & 86 \\
$\mathrm{~F} \rightarrow \mathrm{U} \rightarrow \mathrm{B} \rightarrow \mathrm{K}$ & 85 & $(\mathrm{U}+\mathrm{B}+\mathrm{K}) \rightarrow \mathrm{F}^{\mathrm{c}}$ & 92 \\
$\mathrm{~F} \rightarrow \mathrm{U} \rightarrow \mathrm{K} \rightarrow \mathrm{B}$ & 83 & $(\mathrm{U}+\mathrm{F}+\mathrm{K}) \rightarrow \mathrm{B}^{\mathrm{c}}$ & 85
\end{tabular}




$$
\begin{array}{lll}
\mathrm{F} \rightarrow \mathrm{B} \rightarrow \mathrm{U} \rightarrow \mathrm{K} & 93 & (\mathrm{~B}+\mathrm{F}+\mathrm{K}) \rightarrow \mathrm{U}^{\mathrm{c}} \\
\mathrm{F} \rightarrow \mathrm{B} \rightarrow \mathrm{K} \rightarrow \mathrm{U} & 26 &
\end{array}
$$

${ }^{\mathrm{a}}$ Each stock solution of uracil $(\mathrm{U}), \mathrm{BAO}(\mathrm{B}), \mathrm{K}_{3}\left[\mathrm{Fe}(\mathrm{CN})_{6}\right](\mathrm{F})$ and $\mathrm{KOH}(\mathrm{K})$ was added in the order from the left to the right.

${ }^{\mathrm{b}}$ Two components of the four reagents were mixed and left standing for $10 \mathrm{~min}$ prior to the reaction.

${ }^{\mathrm{c}}$ Three components of the four reagents were mixed and left standing for $10 \mathrm{~min}$ prior to the reaction.

${ }^{\mathrm{d}}$ The RFI obtained by the reaction was taken as 100 , in which the reactants were added in the order of uracil, $\mathrm{BAO}, \mathrm{K}_{3}\left[\mathrm{Fe}(\mathrm{CN})_{6}\right]$ and $\mathrm{KOH}$.

The order in which the reactants were mixed was also seen to affect the results of the reaction (Table 1). After testing 24 combinations, we found that the disordered addition of the reagents weakened the FL intensity, especially when uracil was added last. If any two reactants were mixed and subsequently added to the other two components, there was no diminishing of the FL. However, inhibition of the FL production was observed when BAO, $\mathrm{K}_{3}\left[\mathrm{Fe}(\mathrm{CN})_{6}\right]$ and $\mathrm{KOH}$ were mixed and then incubated prior to the addition of uracil. These results indicated that two major reaction pathways, namely the production of the FL compound and the decomposition of reactants, exist in the present reaction. In order to increase the reaction efficiency, it was better that each reactant was mixed in the order of nucleobase, $\mathrm{BAO}, \mathrm{K}_{3}\left[\mathrm{Fe}(\mathrm{CN})_{6}\right]$ and $\mathrm{KOH}$.

\subsection{Effect of reactants}

BAO (Fig. 3a) was replaced by several BAO-related compounds containing amidoxime functional group. BAO was found to be the most effective fluorogenic reagent, and substitution at any position of phenyl ring suppressed the production of FL (Fig. 3). 4-Trifluoromethylbenzamidoxime (4-trifluoromethyl-BAO) (Fig. 3d) and 4-trifluoromethoxy-BAO (Fig. 3e) generated weaker FL, however, neither 3-trifluoromethyl-BAO (Fig. 3b) nor 3,5-bis(trifluoromethyl)-BAO (Fig. 3c) produced measurable FL. Since amidoxime group is the most reactive moiety in BAO-related compounds, substitution on the phenyl ring would affect the reactivity of amidoxime. The result that formamidoxime (Fig. 3f) did not produce FL suggested that the phenyl ring of BAO is involved in the fluorophore of the product. Non FL was observed when benzamidine (Fig. 3g) was used, whereas benzohydroxamic acid (Fig. 3h) gave a very weak FL. The results demonstrate that the amidoxime group is important to produce the FL substance. A tautomerism of benzohydroxamic acid results in the presence of oxime and hydroxyl groups, which is a similar structure to BAO. This unfavorable tautomerism and weak nucleophilicity of the hydroxyl group would decrease the rate of the conjugation between benzohydroxamic 
acid and uracil. However, even a very small amount of the coupled intermediate might cause a further reaction between the uracil moiety and the amidoxime group, which produced the fluorophore in the same manner as the BAO reaction. Hence the non-fluorogenicity of benzamidine should be attributed to the lack of the reactivity at second stage of the conjugation reaction due to the absence of the amidoxime group. These results indicate that the fluorogenic reagents must have 1) an oxime (or hydroxylamino) group, 2) a nucleophilic functional group next to oxime, and 3) aromaticity. The substitutions on the phenyl ring also affect the fluorogenisity and might break the balance of the reactivity of 1) and 2).
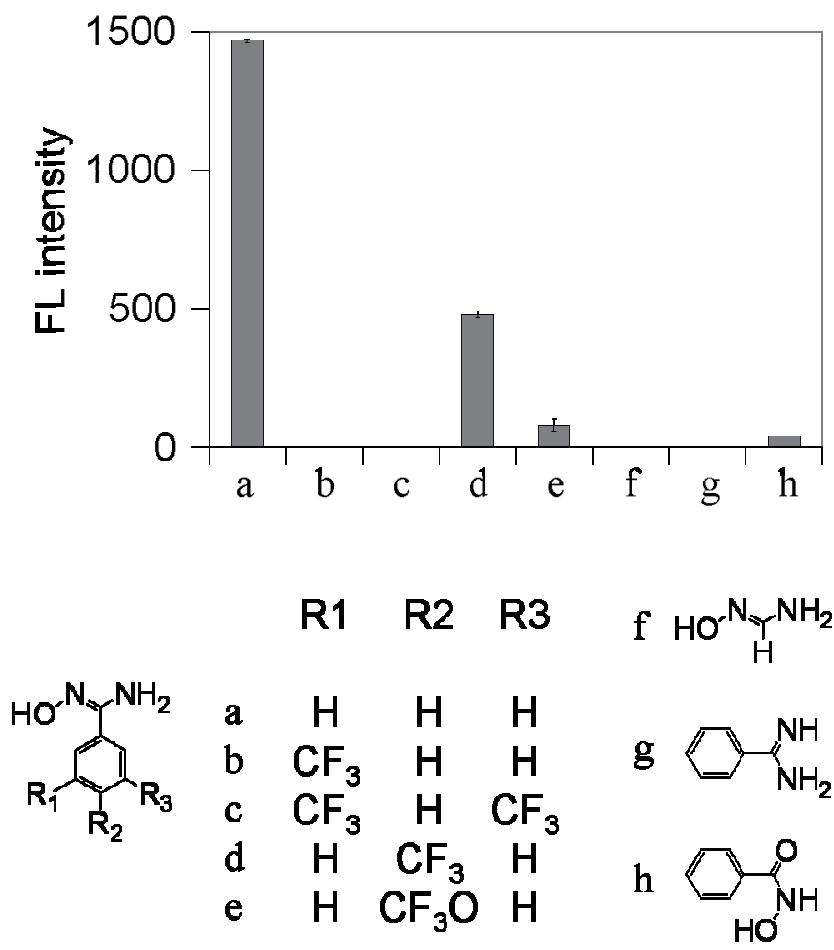

Fig. 3. Reactions of uracil with several BAO-related compounds. (a) BAO, (b) 3-trifluoromethyl-BAO, (c) 3,5-bis(trifluoromethyl)-BAO, (d) 4-trifluoromethyl-BAO, (e) 4-trifluoromethoxy-BAO, (f) formamidoxime, (g) benzamidine and (h) benzohydroxamic acid.

\subsection{Reaction specificity}

The reaction should give a sufficient FL intensity for uracil without any FL intensity for cytosine. According to Fig. 2, the FL production of cytosine was diminished by either increasing the $\mathrm{KOH}$ concentration or shortening the reaction time. Such conditions were obtained when the final concentrations of $1.0 \mathrm{mmol} \mathrm{L}^{-1} \mathrm{BAO}, 2.0 \mathrm{mmol} \mathrm{L}^{-1} \mathrm{~K}_{3}[\mathrm{Fe}(\mathrm{CN})]_{6}$ and $1.0 \mathrm{~mol} \mathrm{~L}^{-1} \mathrm{KOH}$ were adopted and its mixture was heated at $90{ }^{\circ} \mathrm{C}$ for $2 \mathrm{~min}$.

Specificity of this reaction was then checked using other nucleobases (cytosine, thymine, adenine and guanine), nucleosides (2'-deoxyuridine, 2'-deoxycytidine, 2'-deoxythymidine, 
2'-deoxyadenosine and 2'-deoxyguanosine), nucleotides (5'-monophosphate of uridine, cytidine, thymidine, adenosine and guanosine) and several nucleobase analogues (1-methyluracil, 5-fluorouracil, pseudouridine, 6-methyluracil and 5, 6-dihydrouracil). As shown in Fig. 4, uracil only provided FL amongst all substances tested. On the basis of the above results, it is suggested that one or more substitutions at 1-, 5- or 6-position of uracil can disturb the conversion into the FL product, so that uracil-containing nucleosides and nucleotides did not produce the FL compounds. In addition, this reaction did not provide any FL products for 20 kinds of amino acid, sugars (glucose, fructose, lactose, ribose and sucrose), creatine, creatinine and urea. Thus, potential of the present reaction is greatly intense for the accurate quantification of uracil in biological samples without influences from other nucleobases and nucleobase-related components.

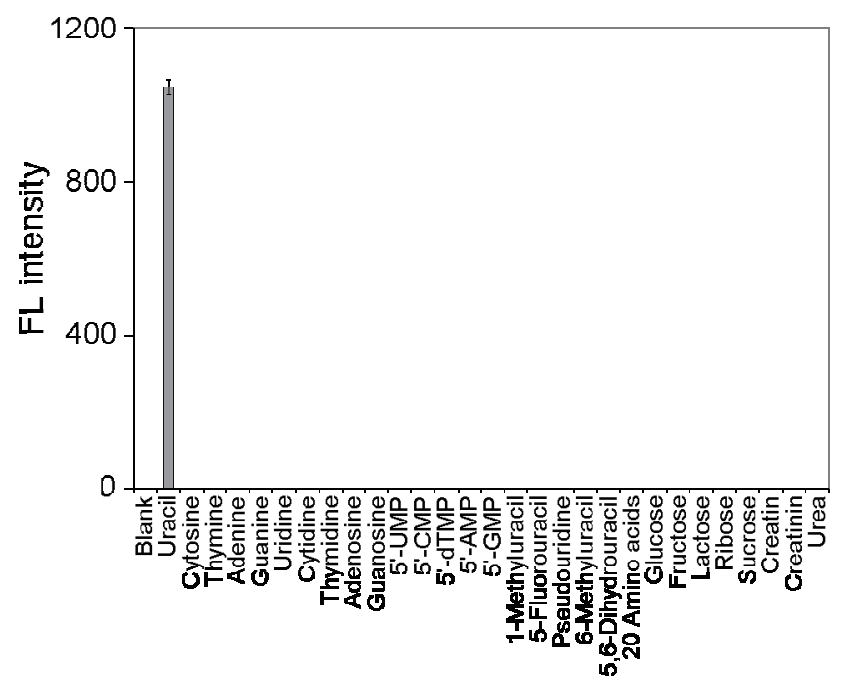

Fig. 4. Reaction specificity of the present method. A final concentration of $250 \mathrm{nmol} \mathrm{mL} \mathrm{m}^{-1}$ of each substance was used except for amino acids (a mixture of 20 amino acids consisted of 10 $\mathrm{mM}$ of each amino acid).

\subsection{Comparative study}

Phenacylbromide is one of few fluorogenic reagents selective for nucleobases [24]. Since no fluorogenic reaction specific for uracil has been reported, the sensitivity of the present BAO reaction was compared with the phenacylbromide reaction using cytosine. The result showed that the BAO reaction with uracil was approximately 400 times more sensitive than the phenacylbromide reaction with cytosine. In case of the phenacylbromide reaction, a linear correlation $\left(\mathrm{Y}=1.44 \times 10^{7} \mathrm{X}+3.28, \mathrm{r}^{2}=0.994\right.$; $\mathrm{Y}$ is the FL intensity and $\mathrm{X}$ is the concentration, mol mL $\mathrm{mL}^{-1}$ of cytosine) between cytosine concentration and FL intensity was obtained up to 2.5

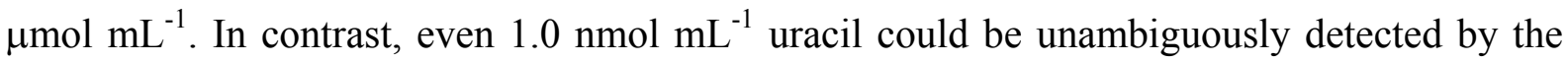
BAO reaction (Fig. 5). The lower detection limit of uracil by the present method was found to 
be approximately $100 \mathrm{pmol} \mathrm{mL}^{-1}(\mathrm{~S} / \mathrm{N}=3)$, and a good linearity $\left(\mathrm{Y}=6.27 \times 10^{9} \mathrm{X}+0.31\right.$, $\mathrm{r}^{2}=0.999$; $\mathrm{Y}$ is the FL intensity and $\mathrm{X}$ is the concentration, $\mathrm{mol} \mathrm{mL}^{-1}$ of uracil). was obtained

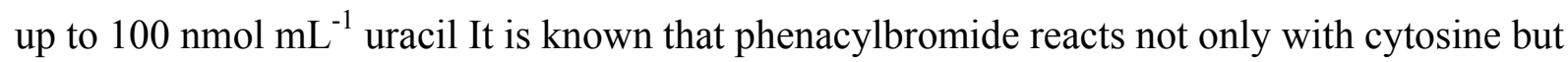
also with adenine and nucleos(t)ides containing cytosine and adenine under heating for 45 min [24]. Therefore, the present FL reaction is rapid and sensitive, possessing an excellent specificity for uracil.

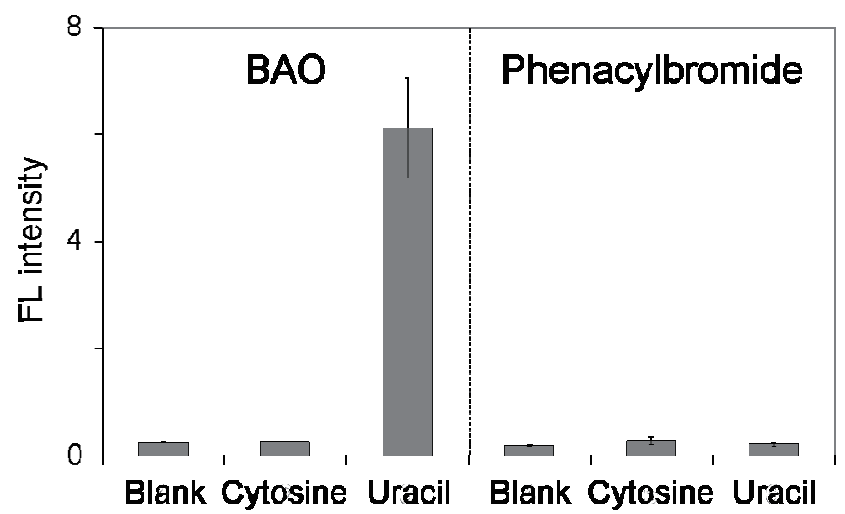

Fig. 5. Comparative study between the reactions of BAO with uracil and of phenacylbromide with cytosine. Nucleobases at $1.0 \mathrm{nmol} \mathrm{mL} \mathrm{mL}^{-1}$ were treated with the BAO reaction $(\mathrm{n}=3$, left panel) and the phenacylbromide reaction ( $n=3$, right panel), respectively. Blank refers to each reaction in the absence of nucleobases.

\subsection{Structural elucidation of FL product}

Our next concern was to determine the chemical structure of the FL product as well as the reaction mechanism. It was found that the FL product by the reaction of uracil with BAO was water-soluble and hard to isolate via extraction from the aqueous solution. Therefore, 4-trifluoromethyl-BAO (Fig. 3d) was used for a large scale synthesis. A single FL product was successfully obtained as a light-blue powder after extraction with ethyl acetate followed by silica gel column chromatographic separation. ${ }^{1} \mathrm{H}-\mathrm{NMR}$ data showed that the product lost two protons at the 5- and 6-positions of uracil, indicating that 4-trifluoromethyl-BAO covalently attached to these positions while maintaining the double bond (data not shown). It is known that the addition of carbonyl group at the $\mathrm{C}^{5}$ of uracil followed by oxidation of the resulting hydroxyl group to a carbonyl group occurs under basic condition in the presence of an oxidant [25]. The initiation of the reaction was thought to be the deprotonation at the $\mathrm{N}^{1}$ and the nucleophilic attack to BAO at the $\mathrm{C}^{5}$. Therefore, substitution at either the $\mathrm{N}^{1}$ or the $\mathrm{C}^{5}$ would inhibit the reaction, and thus this mechanism matches the result that the substitution at the $\mathrm{N}^{1}$ or the $\mathrm{C}^{5}$ did not produce the FL product. There are a couple of reports demonstrating the oxidative cyclization of uracil derivatives containing hydroxiamino or nitroso group in the presence of $\mathrm{K}_{3}\left[\mathrm{Fe}(\mathrm{CN})_{6}\right]$ under basic condition [26, 27]. According to these reactions, intramolecular cyclization most likely happens at the $\mathrm{C}^{6}$ with forming $\mathrm{N}$-oxide, which suits 
the result that the peak corresponding to $-\mathrm{OH}$ in the amidoxime group disappeared. The reason why the reaction with 6-methyluracil did not proceed could be because the substitution at the $\mathrm{C}^{6}$ of pyrimidine ring hinders the nitrogen atom from reaching close proximity due to the planner structure of the intermediate. The predicted structure of the FL product between uracil and 4-trifluoromethyl-BAO is shown in Fig. 6. An ESI-TOF mass spectrum showed strong ion peaks at $\mathrm{m} / \mathrm{z}=297\left([\mathrm{M} \text {-oxigen }+\mathrm{H}]^{+}\right)$, and relatively weak peak at $\mathrm{m} / \mathrm{z}=315$ $\left(\left[\mathrm{M} \text {-oxigen }+\mathrm{H}_{2} \mathrm{O}+\mathrm{H}\right]^{+}\right.$) (data not shown). It is known that the compound containing $\mathrm{N}$-oxide shows a strong $[\mathrm{M} \text {-oxigen }+\mathrm{H}]^{+}$peak [27], which is the same phenomenon as the result obtained in this study. In addition, the FL product by the reaction of uracil with BAO was separated by HPLC (Fig. 2f), and the ESI-TOF mass spectrum was measured. Two main peaks appeared at $\mathrm{m} / \mathrm{z}=229\left([\mathrm{M} \text {-oxigen }+\mathrm{H}]^{+}\right)$and $247\left(\left[\mathrm{M} \text {-oxigen }+\mathrm{H}_{2} \mathrm{O}+\mathrm{H}\right]^{+}\right)$(data not shown) which were both 68 mass less than $\mathrm{m} / \mathrm{z}=297$ and 315 , respectively, obtained by the reaction of uracil with 4-trifluoromethyl-BAO. The number of 68 mass corresponded to the difference of the molecular mass between 4-trifluoromethyl-BAO and BAO. From the above result, the reaction mechanism of uracil with $\mathrm{BAO}$ might be the same as that with 4-trifluoromethyl-BAO.
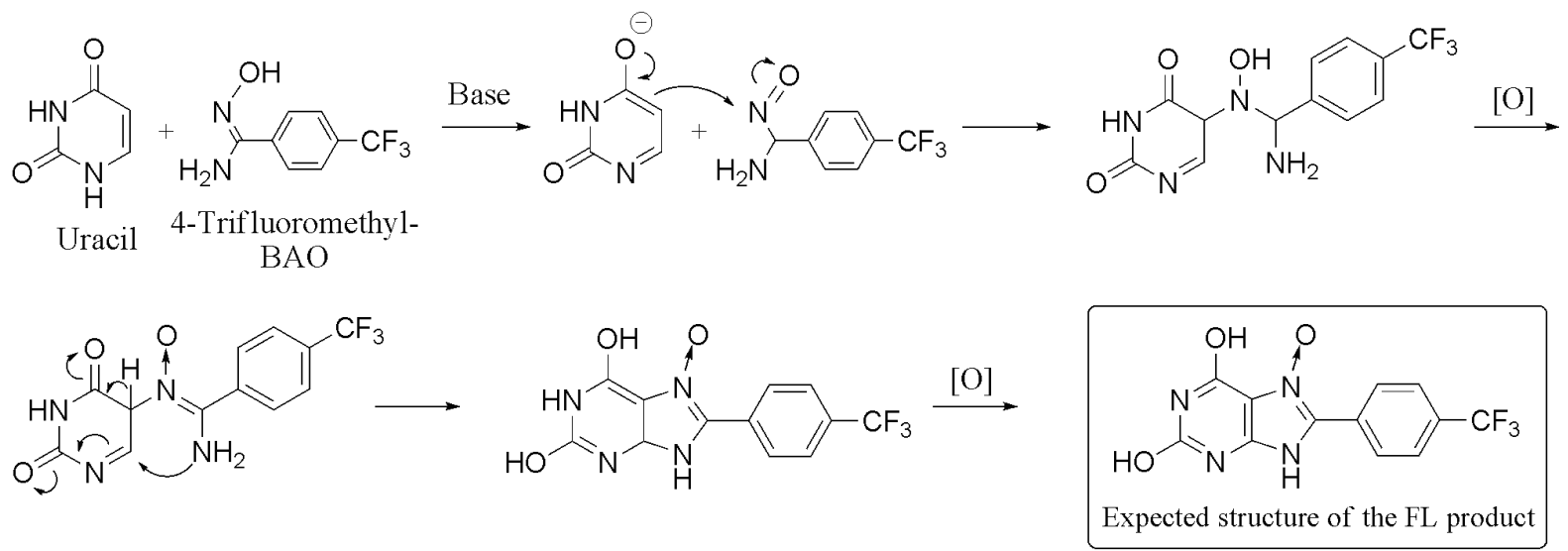

Fig. 6. Plausible reaction mechanism of the fluorogenic reaction between uracil and 4-trifluoromethyl-BAO and expected structure of the FL product.

\section{Conclusions}

In this work, we have developed the novel fluorogenic reaction specific for uracil. The reaction was easily accomplished by heating the mixture of uracil and $\mathrm{BAO}$ in the presence of $\mathrm{K}_{3}\left[\mathrm{Fe}(\mathrm{CN})_{6}\right]$ under basic condition. Although the present system produced weak FL with cytosine, the uracil-specific FL reaction could be achieved by controlling the reaction conditions. The reaction was influenced by the structure of the fluorogenic reagent as well as 
the reaction conditions. According to the analysis of the reaction mechanism, an important factor for the pyrimidine substance to drive the reaction forward is to have no substitution at either $\mathrm{N}^{1}, \mathrm{C}^{5}$ or $\mathrm{C}^{6}$ in the pyrimidine ring and carbonyl group at $\mathrm{C}^{4}$. Taking these things into account, the proposed reaction shows severe structural requirements, indicative of the superior specificity. The reaction gave more than 400 times stronger FL in shorter time as compared with the conventional FL reaction of cytosine with phenacylbromide. These results clearly corroborate that the BAO reaction affords the first example of a uracil-specific fluorogenic reaction, and its highly sensitive and facile protocol should be utilized for further analytical tools such as HPLC, capillary electrophoresis and mass spectrometry. The proposed method will be applied to high-throughput determinations of uracil in urine or blood specimens for a primary screening test of the inborn errors of pyrimidine metabolism such as DPD deficiency.

\section{Acknowledgements}

This work was supported in part by the president's discretionary fund of Nagasaki University, Japan, and partly supported by the Global Center of Excellence Program in Nagasaki University, Japan.

\section{References}

[1] B. Podschun, G. Wahler, K.D. Schnackerz, Eur. J. Biochem. 185 (1989) 219-224.

[2] T.W. Traut, S. Loechel, Biochemistry 23 (1984) 2533-2539.

[3] C. Wasternak, Pharm. Ther. 8 (1980) 629-651.

[4] M.M. Matthews, W. Liao, K.L. Kvalnes-Krick, T.W. Traut, Arch. Biochem. Biophys. 293 (1992) 254-263.

[5] A. Bronckaers, J. Balzarini, S. Liekens, Biochem. Pharmacol. 76 (2008) 188-197.

[6] G.D. Heggie, J.P. Sommadossi, D.S. Cross, W.J. Huster, R.B. Diasio, Cancer Res. 47 (1987) 2203-2206.

[7] D.H. Ho, L. Townsend, M.A. Luna, G..P. Bodey, Anticancer Res. 6 (1986) 781-784.

[8] T.M. Woodcock, D.S. Martin, L.A. Damin, N.E. Kemeny, C.W. Young, Cancer 45 (1980) 1135-1143.

[9] A.P. Lyss, R.C. Lilenbaum, B.E. Harris, R.B. Diasio, Cancer Invest. 11 (1993) 239-240.

[10] H. Okuda, T. Nishiyama, K. Ogura, S. Nagayama, K. Ikeda, S. Yamaguchi, Drug Metab. Dispos. 25 (1997) 270-273.

[11] H. Okuda, K. Ogura, A. Kato, H. Takubo, T. Watabe, J. Pharmacol. Exp. Ther. 287 (1998) 791-799.

[12] T. Inada, T. Jotsuka, G.. Matsuda, H. Kawakubo, Y. Ogata, T. Kubota, Int. J. Clin. Oncol. 4 (1999) 54-56. 
[13] A. Beck, M.C. Etienne, S. Chéradame, J.L. Fischel, P. Formento, N. Renée, G. Milano, Eur. J. Cancer 30 (1994) 1517-1522.

[14] A. Lee, H. Ezzeldin, J. Fourie, R.B. Diasio, Clin. Adv. Hematol. Oncol. 2 (2004) 527-532.

[15] M. Duran, P. Rovers, P.K. de Bree, C.H. Schreuder, H. Beukenhorst, L. Dorland, R.J. Berger, Inherit. Metab. Dis. 14 (1991) 367-370.

[16] S. Sumi, K. Kidouchi, S. Ohba, Y. Wada, J. Chromatogr. B 672 (1995) 233-239.

[17] H. van Lenthe, A.B.P. van Kuilenburg, T. Ito, A.H. Bootsma, A. van Cruchten, Y. Wada, A.H. van Gennip, Clin. Chem. 46 (2000) 1916-1922.

[18] T. Kuhara, C. Ohdoi, M. Ohse, A.B.P. van Kuilenburg, A.H. van Gennip, S. Sumi, T. Ito, Y. Wada, I. Matsumoto, J. Chromatogr. B 792 (2003) 107-115.

[19] B. Podschun, G. Wahler, K.D. Schnackerz, Eur. J. Biochem. 185 (1989) 219-224.

[20] R.A. Fleming, G. Milano, A. Thyss, M-C. Etienne, N. Renée, M. Schneider, F. Demani, Cancer Res. 52 (1992) 2899 -2902.

[21] B.E. Harris, R. Song, S. Soong, R.B. Diasio, Cancer Res. 50 (1990) 197 -201.

[22] M.R. Johnson, J. Yan, L. Shao, N. Albin, R.B. Diasio, J. Chromatogr. B 696 (1997) 183-191.

[23] L.K. Mattison, H. Ezzeldin, M. Carpenter, A. Modak, M.R. Johnson, R.B. Diasio, Clin. Cancer Res. 10 (2004) 2652 -2658.

[24] E.J. Eisenberg, K.C. Cundy, J. Chromatogr. B 679 (1996) 119-127.

[25] D.R. Hwang, P. Helquist, M.S. Shekhani, J. Org. Chem. 50 (1985) 1264-1271.

[26] A.A. Yavolovskii, E.I. Ivanov, Chem. Heterocyc. Compd. 40 (2004) 361-363.

[27] A.A. Yavolovskii, E.I. Ivanov, A.V. Mazepa, Y.E. Ivanov, Russ. J. Gen. Chem. 73 (2003) 1486-1488. 


\section{Figure captions}

Fig. 1. Catabolic pathway of uracil and 5-FU.

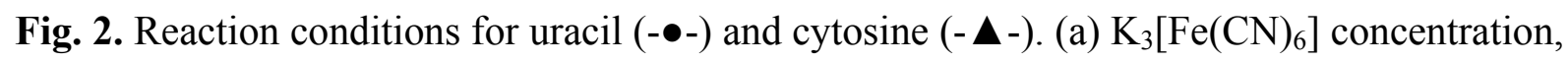
(b) BAO concentration, (c) $\mathrm{KOH}$ concentration, (d) reaction temperature and (e) reaction time. The reaction mixtures with (f) uracil and (g) cytosine were individually separated by HPLC with FL detection.

Fig. 3. Reactions of uracil with several BAO-related compounds. (a) BAO, (b) 3-trifluoromethyl-BAO, (c) 3,5-bis(trifluoromethyl)-BAO, (d) 4-trifluoromethyl-BAO, (e) 4-trifluoromethoxy-BAO, (f) formamidoxime, (g) benzamidine and (h) benzohydroxamic acid.

Fig. 4. Reaction specificity of the present method. A final concentration of $250 \mathrm{nmol} \mathrm{mL} L^{-1}$ of each substance was used except for amino acids (a mixture of 20 amino acids consisted of 10 $\mathrm{mM}$ of each amino acid).

Fig. 5. Comparative study between the reactions of BAO with uracil and of phenacylbromide with cytosine. Nucleobases at $1.0 \mathrm{nmol} \mathrm{mL} \mathrm{mL}^{-1}$ were treated with the BAO reaction $(\mathrm{n}=3$, left panel) and the phenacylbromide reaction ( $n=3$, right panel), respectively. Blank refers to each reaction in the absence of nucleobases.

Fig. 6. Plausible reaction mechanism of the fluorogenic reaction between uracil and 4-trifluoromethyl-BAO and expected structure of the FL product. 